MGM/MGZ wiedergelesen

Johann Chapoutot

\title{
Das Deutsche Kaiserreich am Vorabend des Ersten Weltkrieges - ein »unregierbares Gebilde«?
}

Wolfgang J. Mommsen, Die latente Krise des wilhelminischen Reiches. Staat und Gesellschaft in Deutschland 1890-1914

In: MGM, 15 (1974), 1, S. 7-28; https://doi.org/10.1524/mgzs.1974.15.1.7

https://doi.org/10.1515/mgzs-2017-0158

Wolfgang J. Mommsens Aufsatz ist nicht nur ein brillanter Beitrag zu einer historischen Frage, die Historiker und Öffentlichkeit nach wie vor umtreibt, wie einige erfolgreiche Veröffentlichungen seit 2013 zeigen. Sie ist auch das Zeugnis einer Epoche der Geschichtswissenschaft. Die historiografischen Interessen Mommsens (1930-2004) waren bekanntermaßen vor allem sozialer und politischer Natur. In der Tat greift der Beitrag in den »Militärgeschichtlichen Mitteilungen« (MGM) ein eher klassisches Problem der Sozialgeschichte auf. Doch hinter dem Titel »Staat und Gesellschaft in Deutschland, 1890-1914« verbirgt sich de facto ein Text, wie er für die ziemlich traditionelle Politikgeschichte typisch ist, als hätte diese in den siebziger Jahren angesichts der nahezu unangefochtenen Dominanz der Sozialgeschichte in Deutschland und Frankreich keine volle Daseinsberechtigung (mehr) gehabt oder noch nicht erlangt.

Denn es geht hier sehr wohl um Politikgeschichte: Wolfgang J. Mommsen skizziert die Geschichte der deutschen Reichskanzler unter Kaiser Wilhelm II. von Bismarck bis Bethmann Hollweg und ihr Machtverhältnis zu den anderen Institutionen des Reiches wie dem Reichstag und Bundesrat, zu politischen Kräften wie der Sozialdemokratie und den konservativen Parteien sowie zu sozialen Gruppen wie dem einflussreichen Alldeutschen Verband oder den Kolonialgesellschaften -

Übersetzung: Jörg Echternkamp.

Kontakt: Johann Chapoutot, Université Paris-Sorbonne (Paris IV),

E-Mail: johannchapoutot@hotmail.com

MGZ, (c) 2017 ZMSBw, Potsdam. Publiziert von De Gruyter 
und nicht zu vergessen zum Militär. Dieses Interesse für die Geschichte der Kanzler ist in engem Zusammenhang mit der Epoche zu sehen, in der dieser Aufsatz entstand: Nach der langen Amtszeit von Konrad Adenauer hatte der Wechsel zur Großen Koalition 1966 und anschließend zur sozialliberalen Koalition 1969 unter der charismatischen Führung von Willy Brandt mehr noch als das Grundgesetz von 1949 dazu beigetragen, dass der Kanzler und seine Dienste das politische und historische Interesse auf sich zogen.

Mommsen vertritt in den MGM die These, dass die Macht- und Interessengruppen im Zuge der sozialen und wirtschaftlichen sowie demografischen und geografischen Veränderungen im Deutschland nach den Einigungskriegen von 1866 bis 1871 stark zugenommen hatten. Die Aussage, »dass das Deutsche Reich im Prinzip bereits Anfang der 1890er Jahre ein nahezu unregierbares Gebilde geworden war« (S. 13), habe deshalb ihre Berechtigung. Nicht dass Bismarck nicht bereits vorher Probleme gehabt hätte; das Aufkommen der Sozialdemokratie und der Zentrumspartei bereiteten ihm ernsthafte Schwierigkeiten. Doch nach seiner Entlassung 1890, die Mommsen auf Bismarcks Unfähigkeit zurückführt, die Probleme der Regierung zu lösen (wie Wilhelm II. festgestellt hatte), hatten seine Nachfolger eine umso schwierigere Aufgabe. Mommsen bemerkt hier scharfsinnig, dass die Kanzler von nun an zwischen zwei Machtpolen hin- und hergerissen waren: dem Reichstag, der mit seinem Wahlmodus die Gesellschaft und die neuen sozialen Kräfte, insbesondere die Sozialdemokratie und das liberale Bürgertum einigermaßen angemessen repräsentierte, und dem preußischen Abgeordnetenhaus (Preußen machte Zweidrittel des Reichsgebiets aus), das aufgrund des Dreiklassenwahlrechts in einem Konservatismus gefangen war, der sich mit der Zeit eher noch verstärkte. Der Kanzler musste versuchen, zwischen diesen beiden Instanzen ein zweifelhaftes Gleichgewicht zu finden. Er musste außerdem Zugeständnisse an die verschiedenen politischen und sozialen Kräfte machen, die im neuen Reich immer mehr an Bedeutung gewannen: Neben den politischen Parteien, die ihre Macht behaupteten (SPD und Zentrum), demonstrierten auch die Industrie, das Militär und die verschiedenen Interessenverbände (Alldeutscher Verband, Flottenverein etc.) ihre Stärke.

Mommsen unterscheidet sehr klar zwischen zwei Arten von Regierungserfahrungen in den Jahren nach Bismarck: zum einen die liberale Regierung Caprivis, die eine innen- wie außenpolitische Befriedung anstrebte, und zum anderen die Regierung Bülow, die auf beiden Gebieten offensiver vorging. Bismarcks Nachfolger Caprivi legte sein Amt 1894 nieder, weil er das autoritäre Machtstreben von Wilhelm II., von Industriekreisen und vom Militär angesichts der wachsenden Sozialdemokratie ablehnte. Was Bülow betraf, so spielte dieser ein Machtspiel mit den Feinden des Konservatismus im Inneren und den Widerständlern gegen die wachsende Macht Deutschlands im Ausland. Diese Politik war zwar nicht von 
Misserfolgen, wohl aber doch von Aufsehen erregenden Fiaskos geprägt. Dazu gehörte u.a. die Daily-Telegraph-Affäre von 1908.

Bülows Nachfolger, Bethmann Hollweg, wagte eine Politik des Kompromisses, die nahezu alle - mit Ausnahme der Sozialdemokraten - zufriedenstellen sollte. Mommsen merkt an, dass unter der Regierung von Bethmann Hollweg die strukturellen und konstitutionellen Mängel des Reiches am deutlichsten zu Tage traten: Bethmann Hollweg versuchte, den inneren und äußeren Frieden aufrechtzuerhalten, was ihm jedoch nicht gelang, da er Zugeständnisse an alle machen musste. Zwei Ereignisse zeigen seine Ohnmacht: die Zabern-Affäre 1913, in der die Regierung Bethmann Hollweg für die Armee Partei ergriff, obwohl diese eindeutig schuldig war; und der Durchbruch der Sozialdemokraten, die als Sieger aus den Reichstagswahlen 1912 hervorgingen. Der Kanzler wollte »Zeit gewinnen« (S. 22), doch die nationale und internationale Lage ließ ihm dazu kaum die Möglichkeit.

Darüber hinaus kam es durch die Lähmung des Reichstags, in dem sich mehrere Parteien unversöhnlich gegenüberstanden und der von der SPD dominiert wurde, zu einer Verlagerung des Spiels der politischen Kräfte in den außerparlamentarischen Raum (S. 23), in dem militärische, industrielle, pangermanische und kolonialistische Interessenverbände immer mehr auf die Öffentlichkeit sowie das Umfeld des Kaisers einwirkten. Laut Mommsen übernahmen diese »Agitationsverbände« - der Alldeutsche Verband, der Deutsche Wehrverein und der Flottenverein - immer mehr die »politische Initiative« (S. 23), indem sie durch intensive Propaganda auf eine Öffentlichkeit einwirkten, die sich von einer bestenfalls »tolerierten « Minderheitsregierung nicht länger repräsentiert fühlten.

Es bleibt jedoch festzuhalten, dass die Leitung der Außenpolitik des Reiches ein großes Privileg der Regierung und des Kanzlers war. Bethmann Hollwegs hohes Ansehen im Ausland war für ihn ein Garant für Stabilität: Wilhelm II. dagegen wusste um seinen schlechten Ruf außerhalb Deutschlands und konnte sich deshalb nicht von seinem Kanzler trennen, auch wenn die beiden unterschiedlicher Ansicht darüber waren, mit welcher Entschlossenheit man den »Feinden des Reiches« begegnen sollte.

Bethmann Hollweg widersetzte sich dem Druck der äußerst nationalistischen und militaristischen Kreise und lehnte den Gedanken eines Präventivkrieges ab, insbesondere gegen Russland, dessen Generalstab befürchtete, dass Russland seit 1916/1917 nicht mehr hinreichend militärisch gerüstet ist. Die Vorstellungen, die sich der Kanzler von den internationalen Beziehungen und vom Völkerrecht machte, verboten ihm eine solch radikale Perspektive. Es gelang ihm laut Mommsen, den Kaiser davon zu abzubringen. Das Zitat, das Mommsen anführt, um die Argumente des Kanzlers zusammenzufassen, ist insofern verblüffend, als es zeigt, in welchem Maße Bethmann Hollweg ein scharfes Bild der politischen Kräfteverhältnisse im Inneren des Deutschen Reiches zeichnet: In seinen Augen würde ein 
Krieg nur der Sozialdemokratie zum Vorteil gereichen und am Ende zum Zusammenbruch des Kaiserreiches führen (S. 25).

Hier zeigt sich laut Mommsen am deutlichsten die Unfähigkeit der deutschen Regierung, die außenpolitische Lage zu bewältigen, da sie nicht einmal die Widersprüche im Inneren überbrücken kann. Der Kaiser und sein Kanzler sind sich zwar einig, was den Verzicht auf einen Präventivkrieg und die Gefahren, die ein solcher Konflikt mit sich bringen würde, betrifft. Doch dies ändert nichts an der Tatsache, dass die auf der internationalen Bühne seit Langem isolierte deutsche Regierung in ihrer Balkanpolitik auf absolute Loyalität und nahezu bedingungslose Solidarität mit Österreich-Ungarn setzt, insbesondere nach dem Attentat von Sarajewo.

Mommsen geht nur kurz auf die österreichische Politik ein, da sie nicht Gegenstand seines Aufsatzes ist. Ihm zufolge stieß diese Politik, die sich seit den 1870er Jahren zunehmend expansionistisch gestaltete, auf heftigen Widerstand der Nationalisten, der seinen tragischsten Ausdruck im Attentat von Sarajewo fand. Konnte sich Wien zunächst noch als Opfer sehen, so wurde die österreichische Politik gegenüber Serbien im Juli 1914 problematisch, ja sogar katastrophal. Mommsen sagt dazu an dieser Stelle nichts. Er beschränkt sich auf die Feststellung, dass die deutsche Regierung in Person ihres Kanzlers bei einer Entwicklung (dem Krieg) enttäuscht zusehen musste, die der Kanzler gewiss nicht gewollt, die er aber auch sicherlich nicht zu verhindern gewusst hatte oder hätte verhindern können: Man habe die Richtung verloren und der Stein sei ins Rollen gekommen, erklärte der Kanzler am 29. Juli 1914 - zu einem Zeitpunkt, an dem wohlgemerkt der Frieden noch möglich war (S. 28).

Wolfgang J. Mommsens Aufsatz ist in dreierlei Hinsicht interessant. Erstens handelt es sich, wie einleitend erwähnt, um einen politikgeschichtlichen Aufsatz im klassischen Sinne, der in einer Zeit des erdrückenden Paradigmas der Sozialgeschichte an den deutschen Universitäten nicht in vollem Umfang als solcher bezeichnet wurde. Sicherlich findet hier die Gesellschaft in Form von Kräften, die auf die politischen Entscheidungen der deutschen Regierung einwirken, Erwähnung, doch de facto handelt sich um eine politikgeschichtliche Arbeit.

Zweitens ist diese Arbeit interessant, weil hier sämtliche Elemente der klassischen Politikgeschichte entfaltet werden, um sie auf außenpolitische Fragen anzuwenden. Der Historiker greift auf klassische Quellen der Politikgeschichte zurück: Augenzeugenberichte, Memoiren, Parlamentsprotokolle, Sitzungsprotokolle des Kabinetts etc. Im Übrigen beherrscht Mommsen die Geschichtsschreibung in jeder Hinsicht. Die Methodologie stammt aus dem Bereich der Politikgeschichte in ihrer traditionellsten Ausprägung: Welche Kräfte wirken im Rahmen der bestehenden Institutionen? Wie beeinflussen und bestimmen sie die politische Entscheidungsfindung? Diese Fragen werden hier gestellt. 
Drittens ist Mommsens Beitrag in den MGM von Interesse, da er diese Frage im Hinblick auf ein bestimmtes Thema stellt: den Weg zum Krieg und die Verantwortung Deutschlands für den Kriegsausbruch. Dieser Aufsatz wurde 1974 veröffentlicht, das heißt sechzig Jahre nach Kriegsausbruch. Im Übrigen erschien elf Jahre zuvor Fritz Fischers Buch »Griff nach der Weltmacht« (1963), in dem der Hamburger Historiker Deutschland eine eindeutige Verantwortung für den Ausbruch des Krieges zuschreibt. Fischer entwickelt in seinem Buch die These vom deutschen Sonderweg in ihrer klassischsten Form: Deutschland ist eine imperialistische Macht, die aus demografischen, wirtschaftlichen und geostrategischen Gründen versucht, ihr Gebiet seit 1914 gewaltsam zu erweitern. Diese These war in den 1950er und 1960er Jahren im Bereich der Sozial-, Militär-, Politik- und Wirtschaftsgeschichte weithin akzeptiert: Ein politisch rückständiges, aber wirtschaftlich und demografisch modernes Deutschland habe demnach versucht, seine Widersprüche im Inneren durch den Krieg zu überbrücken.

Mommsen widerspricht dieser These auf subtile Weise, ohne es ausdrücklich zu sagen. Nein, Deutschland war politisch nicht so archaisch wie allgemein behauptet. Die gesellschaftlichen Kräfte wurden in die Machtzirkel aufgenommen und beeinflussten die politische Entscheidungsfindung. Angesichts der älteren Sicht des politischen Systems im Kaiserreich war es paradoxerweise ein übertriebener Liberalismus, der zur Unentschlossenheit und Ohnmacht angesichts der von den deutschen politischen Kräften geäußerten Widersprüche geführt hatte. Die Statistenrolle des Kanzlers, der nur noch Zuschauer des im Sommer 1914 vorwiegend von Wien inszenierten großen europäischen Dramas war, ist ein eindringlicher Beweis für diese Demonstration.

Der Titel des Aufsatzes lässt seine ganze Bedeutung erst nach der Lektüre des Textes erkennen. Es handelt sich hier tatsächlich um »die latente Krise des wilhelminischen Reiches « - eine latente Krise der Institutionen, die im Sommer 1914 in Form einer Starre offen zu Tage tritt. Diese Lähmung der regierenden Mächte widerspricht allen gängigen Stereotypen vom »ruhelosen Reich« (Michael Stürmer), dessen politische Führung angeblich genauso kriegslustig war wie seine Bevölkerung. Mommsen beschreibt hier nicht eine deutsche Regierung, die entschlossen war zu kämpfen, sondern schildert die Missgeschicke einer schwachen Macht, die es mit Widersprüchen in der deutschen Gesellschaft sowie der Komplexität der politischen Institutionen als Folge eines zugleich regionalistischen und liberalen Kompromisses zwischen 1866 und $1871 \mathrm{zu}$ tun hatte. Es verblüfft, dass Wolfgang J. Mommsens Bruder, Hans Mommsen, zur gleichen Zeit, Anfang der 1970er Jahre, Hitler als einen »schwachen Diktator« beschreibt. Die 1960er und 1970er Jahre, die von Protesten der politischen und studentischen Kreise gegen die etablierten Mächte geprägt waren, waren auch jene Jahrzehnte, in denen im Bereich der Geisteswissenschaften die Macht einzelner Personen 
radikal infrage gestellt wurde. Man denke nur an Michel Foucault und Pierre Bourdieu, die von »Disziplin « bzw. »Beherrschung " sprachen und Prozesse meinten, die komplexer waren als die Beziehung zwischen oben und unten, auf die der Begriff »Macht« hinweist.

Mommsen, der sich hier als führend im Bereich der Epistemologie der Geisteswissenschaften erweist, zeigt sich somit im Widerspruch zur Geschichtsschreibung seiner Zeit und zum Mainstream der Sonderwegsthese, welche die nationalsozialistische Gewalt auf die wilhelminischen Frustrationen zurückführte. Er sagt es nicht ausdrücklich, weil das seine derzeitige und spätere akademische Stellung hätte gefährden können. Doch dem aufmerksamen Leser entgeht dieser Widerspruch nicht. Im Übrigen greifen seine Schlussfolgerungen zur Rolle (oder nicht vorhandenen Rolle) Berlins im Sommer 1914 den jüngsten Erkenntnissen von Christopher Clark vor, der in seinem viel diskutierten und überzeugenden Buch die deutsche Regierung als einen Nebenakteur in der europäischen Krise von 1914 darstellt, während er Österreich-Ungarn die Hauptrolle zuweist.

Der Nutzen einer erneuten Lektüre dieses MGM-Aufsatzes von Wolfgang J. Mommsen ist gar nicht zu unterschätzen. Auf einer ersten Betrachtungsebene zeigt sich, dass hier ein historisches Verfahren gekonnt angewandt wird und zu wichtigen Erkenntnissen für die Geschichte des Kaiserreiches führt. Auf einer zweiten Ebene ist sein Platz in der Geschichtsschreibung unbestritten, obwohl die hier aufgeworfenen Fragen (die Macht des Kanzlers, die Verantwortung der deutschen Regierung für die Krise 1914) in jeder Hinsicht von der politischen, historiografischen und epistemologischen Aktualität seiner Zeit bestimmt werden. Mommsens Text erweist sich daher in dreifacher Hinsicht als zeitgeschichtlich: ein Aufsatz der Zeitgeschichte, der als Text seiner Zeit auch heute aktuell ist. Das macht ihn möglicherweise aus, einen Klassiker. 